Working Paper 92-08

February 1992
División de Economía

Universidad Carlos III de Madrid

Calle Madrid, 126

28903 Getafe (Madrid)

A NOTE ON THE MULTIVARIATE BOX-COX

TRANSFORMATION TO NORMALITY

\author{
Santiago Velilla*
}

\title{
Abstract
}

We study some aspects of the multivariate Box-Cox transformation to normality which have received little attention in the literature.

Key words

Asymptotic relative efficiency (ARE); Concentrated log-likelihood; Multivariate BoxCox transformation.

* Departamento de Estadística y Econometría, Universidad Carlos III de Madrid. 28903-Getafe (Madrid), Spain. 


\section{A NOTE ON THE MULTIVARIATE BOX-COX TRANSFORMATION TO NORMALITY}

\section{Santiago Velilla}

Departamento de Estadistica, Universiclad Carlos III de Madrid, 28903-Getafe (Madrid), Spain.

Alestract: We study some aspects of the multivariate Box-Cox transformation to normality which have received little attention in the literature.

Key wards: Asymptotic relative efficiency (ARE); Concentrated log-likelihood; Multivariate Box-Cox transformation.

\section{INTRODUCTION}

Let $\mathrm{X}$ be a random variable which takes values denoted by $\mathrm{x}$. If the distribution of $\mathrm{X}$ is not normal, it is sometimes convenient to consider transformations that help to normalize the observed data. When $x>0, a$ useful family of transformations is the family of Box and Cox (1964):

$$
x^{(\lambda)}= \begin{cases}\frac{x^{\lambda}-1}{\lambda}, & \lambda \neq 0 ; \\ \log x, & \lambda=0 .\end{cases}
$$

Note that the transformation in (1) is indexed by the scalar parameter $\lambda$.

Consider now a $\mathrm{p}$-variate random vector $\mathrm{X}=\left(\mathrm{X}_{1}, \ldots, \mathrm{X}_{\mathrm{p}}\right)^{\prime}$ such that all its components take positive values. When the distribution of $\mathbf{X}$ is 
not multivariate normal, Andrews et al. (1971) have given the following generalization of (1). We have a p-vector $\Lambda=\left(\lambda_{1}, \ldots, \lambda_{p}\right)^{\prime}$ of transformation parameters, one for each dimension, such that the model

$$
x^{(\Lambda)}=\left(x_{1}^{(\lambda)}, \ldots, x_{p}^{\left(\lambda_{p}\right)}\right)^{\prime} \sim N_{p}(\mu, \Sigma)
$$

holds. We will write the parameters in model (2) in the form $\Theta=(\Lambda, \mu, \Sigma)$, where $\mu=\left(\mu_{1}, \ldots, \mu_{p}\right)^{\prime}$ and $\Sigma=\left(\sigma_{1 j}\right)_{\text {pxp }}$.

With few exceptions (see, for example, section 5.3 in the book by Gnanadesikan (1977) or section 4.2 in the book by Seber (1984)), the multivariate Box-Cox transformation has received little attention in the literature. Computational and inferential procedures remain to be explored. In this note we derive, in section 2 , a result which is shown to be useful for computational purposes. In section 3, we propose a general methodology for making inferences about the parameter $\Lambda$. Finally, in section 4 , we study some efficiency properties of the MLE estimator $\hat{\Lambda}$ of $\Lambda$ in model (2).

\section{A COMPACT EXPRESSION FOR THE CONCENTRATED LOG-LIKELIHOOD FOR $\Lambda$}

Let $x=\left(x_{1 j}\right)=\left(x_{1}, \ldots, x_{p}\right)$ a nxp data matrix from model (2) and assume that the rows of the transformed data matrix $x^{(\Lambda)}$, namely, $\mathbf{x}_{1}^{(\Lambda)}=\left(x_{11}^{\left(\lambda_{1}\right)}\right.$, $\left.\ldots, x_{1 p}^{(\lambda)}\right), \quad i=1, \quad \ldots, n$, are i.i.d. $\quad N_{p}(\mu, \Sigma)$. If $L(\Theta)=L(\Lambda, \mu, \Sigma)$ represents the associated log-likelihood, standard normal theory shows that the concentrated log-likelihood $\mathrm{L}_{\max }(\Lambda)$ for the transformation parameter is (up to an additive constant),

$$
L_{\max }(\Lambda)=-(n / 2) \log \left[\left|x^{(\Lambda)^{\prime}}\left(I_{n}-1_{n} 1_{n}^{\prime} / n\right) X^{(\Lambda)}\right|\right]+\log \left[\Lambda_{\Lambda}(X)\right] \text {, }
$$

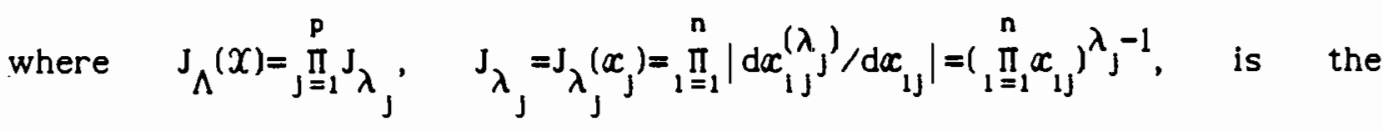
jacobian of the transformation. Define, for each column $j$ of the data matrix $x, \quad j=1, \quad \ldots, p$, the $n$ vector of normalizad variables 


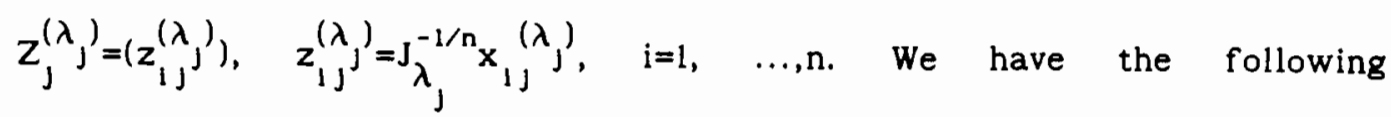
result.

LEMMA 2.1. Let $z^{(\Lambda)}=\left(z_{1}^{\left(\lambda_{1}\right)}, \ldots, z_{p}^{\left(\lambda_{p}\right)}\right)$ be the nxp matrix of normalized variables. The concentrated $\log$-likelihood for $\Lambda$ is (up to an additive constant)

$$
L_{\max }(\Lambda)=-\frac{n}{2} \log \left[\left|Z^{(\Lambda)^{\prime}}\left(I_{n}-1_{n} I_{n}^{\prime} / n\right) Z^{(\Lambda)}\right|\right]
$$

PROOF. Define $\quad \mathrm{D}=\operatorname{diag}\left(\mathrm{J}_{\lambda_{1}^{1 / n}}^{1 / \mathrm{n}}, \quad \ldots, \quad \mathrm{J}_{\lambda_{\mathrm{p}}}^{1 / \mathrm{n}}\right)$ so that $-(n / 2) \log \left[J_{\Lambda}^{-2 / n}(x)\right]=-(n / 2) \log \left[|D|^{-2}\right]$. Taking account that $z^{(\Lambda)}=x^{(\Lambda)} D^{-1}$ and recalling expression (3), the result (4) follows.

For computational purposes, expression (4) shows that the MLE estimator $\hat{\Lambda}$ is obtained by minimizing in $\Lambda$ the determinant

$$
\left|z^{(\Lambda)^{\prime}}\left(I_{n}-1_{n} 1_{n}^{\prime} / n\right) z^{(\Lambda)}\right|
$$

which depends on the nxp matrix $z^{(\Lambda)}$. It is easily seen that expression (5) generalizes the minimization problem associated with the determination of the MLE of $\lambda$ in the scalar case of model (1). Recall that the $(i, j)$ element of the determinant in (5) is of the form $\sum_{k=1}^{n}\left(z_{k 1}^{\left(\lambda_{1}\right)}-\bar{z}_{1}^{\left(\lambda_{1}\right)}\right)\left(z_{k j}^{\left(\lambda_{j}\right)}-\bar{z}_{j}^{\left(\lambda_{j}\right)}\right), \quad$ where $\quad \bar{z}_{1}^{\left(\lambda_{1}\right)}=(1 / n) \sum_{k=1}^{n} z_{k 1}^{\left(\lambda_{1}\right)} \quad$ and $\bar{z}_{j}^{\left(\lambda_{j}\right)=(1 / n)} \sum_{k=1}^{n} z_{k j}^{\left(\lambda_{j}\right)}, \quad i, j=1, \ldots, p$. In principle, minimization of (5) can be carried out by using the appropriate numerical subroutine. However, it is common to encounter computing overflow problems in the determination of the jacobian $J_{\Lambda}(x)$, particularly when $n$ is large. We can use lemma 2.1 above to derive the following invariance property of $\hat{\Lambda}$ which is useful for overcoming these inconveniences. 
THEOREM 2.1. Let $S=\operatorname{diag}\left(s_{1}, \ldots, s_{p}\right)$, where $s_{j}>0, j=1, \ldots, p$, and consider the scaled data matrix $\tilde{x}=x \mathrm{~S}^{-1}$. Write, in obvious notation, the corresponding matrix $\tilde{\mathbf{Z}}^{(\Lambda)}=\left(\tilde{\mathrm{Z}}_{1}^{\left(\lambda_{1}\right)}, \ldots, \tilde{\mathrm{Z}}_{\mathrm{p}}^{\left(\lambda_{\mathrm{p}}\right)}\right)$. We have,

$$
\left|\tilde{\mathbf{z}}^{(\Lambda)^{\prime}}\left(I_{n}-1_{n} 1_{n}^{\prime} / n\right) \tilde{\mathbf{Z}}^{(\Lambda)}\right|=|S|^{-2}\left|\mathbf{Z}^{(\Lambda)^{\prime}}\left(I_{n}-1_{n} l_{n}^{\prime} / n\right) Z^{(\Lambda)}\right| .
$$

PROOF. Write $\tilde{x}=\left(\tilde{x}_{1}, \ldots, \tilde{x}_{p}\right)$ so that $\tilde{x}_{j}=x_{j} / s_{j}$. Let $q_{j}=-s_{j}^{-1} J_{\lambda}^{-1 / n}\left(x_{j}\right) s_{j}\left(\lambda_{j}\right)$, $\mathrm{j}=1, \quad \ldots, \mathrm{p}$. Using $J_{\lambda j}\left(\tilde{x}_{j}\right)=\left(\prod_{1=1}^{n} \tilde{x}_{1 j}\right)^{\lambda} j_{j}^{-1}=s_{j}^{n\left(1-\lambda_{j}\right)_{J}} \lambda_{j}\left(x_{j}\right), \quad$ it $\quad$ is not difficult to show that $\left.\tilde{z}_{1 j}^{(\lambda)}\right)^{\prime}=\lambda_{j}^{-1 / n}\left(\tilde{x}_{j} \tilde{x}_{1 j}^{\left(\lambda_{j}\right)}=s_{j}^{-1} z_{1 j}^{(\lambda)}+q_{j}\right.$. Therefore, we have

$$
\tilde{\mathbf{z}}^{(\Lambda)}=\mathrm{Z}^{(\Lambda)} \mathrm{S}^{-1}+\mathrm{l}_{\mathrm{n}} \mathrm{Q}^{\prime},
$$

where $Q$ is the $p \times 1$ vector $Q=\left(q_{1}, \ldots, q_{p}\right)^{\prime}$. Expression (6) follows directly from (7) by observing that $\left(I_{n}-1 l_{n} 1^{\prime} / n\right) I_{n}=0$.

As a consecuence of (6), the determination of $\hat{\Lambda}$ is not affected by scaling appropriately the data matrix $x$ with a collection of constants \{s\} not depending on $\Lambda$. For example, in practical applications with positive data, a convenient choice of the jth scale factor is $s_{j}=\max _{1 \leq 1 \leq n} x_{1,}, j=1, \ldots, p$, so that the modified data matrix $\tilde{x}$ has all its entries between 0 and 1 .

\section{INFERENCE ON THE TRANSFORMATION PARAMETER $\wedge$}

Under model (2), we can obtain, on the basis of asymptotic considerations, that the set of all $\Lambda$ such that

$$
2\left[L_{\max }(\hat{\Lambda})-L_{\max }(\Lambda)\right] \leq \chi_{p, \alpha^{2}}^{2}
$$


where $\chi_{p, \alpha}^{2}$ is the upper $100 \alpha \%$ point of a chi-squared distribution with $p$ degrees of freedom, is an approximate $(1-\alpha) \times 100 \%$ confidence region for $\Lambda$. This is the methodology proposed by Andrews et al. (1971) for making inferences about $\Lambda$ (see also Gnanadesikan (1977, sec. 5.3)). In this section we propose an alternative approach based on the expression (4) for $L_{\max }(\Lambda)$.

Under suitable regularity conditions, we have that

$$
\hat{\Lambda} \sim \mathrm{AN}_{\mathrm{P}}[\Lambda,(1 / \mathrm{n}) \mathrm{J}(\Lambda, \Lambda)] \text {, }
$$

where $J(\Lambda, \Lambda)$ is the corresponding block for the parameter $\Lambda$ in the inverse of the information matrix $I(\Theta)=-E\left[\partial / \partial \theta(\partial L(\Theta) / \partial \Theta)^{\prime}\right]$ under model (2). A general method for obtaining an estimate for $(1 / n) J(\Lambda, \Lambda)$, as exposed, for example in Seber and Wild (1989, p. 38), is to compute

$$
-\left[\left.\frac{\partial}{\partial \Lambda}\left(\frac{\partial}{\partial \Lambda} L_{\max }(\Lambda)\right)^{\prime}\right|_{\Lambda=\hat{\Lambda}}\right]^{-1}
$$

The main problem in dealing with (10) lies in finding the expression for the second partial derivatives $\partial^{2} L_{\max }(\Lambda) / \partial \lambda_{r} \partial \lambda_{s}, r, s=1, \ldots, p$, at $\Lambda=\hat{\Lambda}$. These are obtained as follows.

Write $M(\Lambda)=Z^{(\Lambda)^{\prime}}\left(I_{n}-11_{n}^{\prime} / n\right) Z^{(\Lambda)}$ and define, for each $j=1, \ldots, P$,

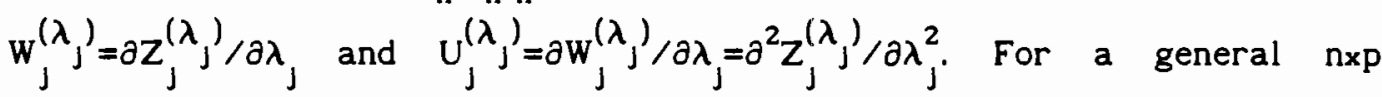
matrix $H=\left(h_{1}, \ldots, h_{p}\right)$, we will write $H_{j}(u)$ for the $n x p$ matrix obtained from $H$ by replacing its jth column by the $n \times 1$ vector $u$. We will also write $H_{j k}(u, v)$ for the $\mathrm{nxp}$ matrix obtained replacing its jth and $k$ th column by, respectively, $u$ and $v$. If we put $Z_{j}, w_{j}$ and $U_{j}$ for the corresponding functions $z_{j}^{\left(\lambda_{j}\right)}, w_{j}^{\left(\lambda_{j}\right)}$ and $U_{j}^{\left(\lambda_{j}\right)}$ evaluated in the jth coordinate of the MLE estimator $\hat{\Lambda}$, we have the following result.

THEOREM 3.1. Let $Z=Z^{(\hat{\Lambda})}=\left(Z_{1}, \ldots, Z_{p}\right)$ and $A=\left(I_{n}-1_{n} 1_{n}^{\prime} / n\right)$. We have: 


$$
\begin{aligned}
& \text { a) } \partial L_{\max }(\Lambda) /\left.\partial \lambda_{r}|\Lambda=\hat{\Lambda}=-n| M(\hat{\Lambda})\right|^{-1}\left|Z_{r}^{\prime}\left(W_{r}\right) A Z\right| ; \\
& \text { b) } \partial^{2} L_{\max }(\Lambda) /\left.\partial \lambda_{r}^{2}|\Lambda=\hat{\Lambda}=-n| M(\hat{\Lambda})\right|^{-1}\left[\left|Z_{r}^{\prime}\left(W_{r}\right) A Z_{r}\left(W_{r}\right)\right|+\left|Z^{\prime} A Z_{r}\left(U_{r}\right)\right|\right]
\end{aligned}
$$

for $r=1, \ldots, p$. We also have:

$$
\text { c) } \partial^{2} L_{\max }(\Lambda) /\left.\partial \lambda_{r} \partial \lambda_{s}|\Lambda=\hat{\Lambda}=-n| M(\hat{\Lambda})\right|^{-1}|| Z_{r s}^{\prime}\left(W_{r}, W_{s}\right) A Z|+| Z_{r}^{\prime}\left(W_{r}\right) A Z_{s}\left(W_{s}\right) \mid l
$$

for $r, s=l, \ldots, p(r \neq s)$.

The proof of this theorem is based on the following lemma.

LEMMA 3.1. Let $\mathrm{C}, \mathrm{D}$, and $\mathrm{B}$ be three $\mathrm{nxp}$ matrices and let $\mathrm{E}$ be $\mathrm{a}$ symmetric matrix of $n \times n$. If $e_{1}$ represents the ith canonical vector of $\mathbb{R}^{n}$, we have, for $i=1, \ldots, n, j=1, \ldots, p$, the following differentiation formulae:

$$
\begin{aligned}
& \text { a) } \left.\partial\left|C^{\prime} E D\right| / \partial c_{1 j}=\left|C_{j}^{\prime}\left(e_{1}\right) E D\right| ; b\right) \partial\left|C^{\prime} E D\right| / \partial d_{1 j}=\left|C^{\prime} E D_{j}\left(e_{1}\right)\right| ; \\
& \text { c) } \partial\left|B^{\prime} E B\right| / \partial b_{1 j}=2\left|B_{j}^{\prime}\left(e_{1}\right) E B\right| .
\end{aligned}
$$

PROOF. See appendix.

PROOF OF THEOREM 3.1. a) By the chain rule and part c) of lemma 3.1 above, we have

$$
\begin{aligned}
& \partial \mathrm{L}_{\max }(\Lambda) /\left.\partial \lambda_{r}|\Lambda=\hat{\Lambda}=-\mathrm{n}| \mathrm{M}(\hat{\Lambda})\right|^{-1} \sum_{i=1}^{n} \sum_{j=1}^{\mathrm{p}}\left|\mathbf{Z}_{j}^{\prime}\left(e_{1}\right) \mathrm{AZ}\right|\left(\partial \mathbf{z}_{1 j}^{\left(\lambda_{j}\right)} /\left.\partial \lambda_{r}\right|_{\Lambda=\hat{\Lambda}}\right) \\
& =-n|M(\hat{\Lambda})|^{-1} \sum_{l=1}^{n}\left|Z_{r}^{\prime}\left(e_{1}\right) A Z\right| w_{i r} \\
& =-n|M(\hat{\Lambda})|^{-1} \sum_{l=1}^{n}\left|Z_{r}^{\prime}\left(w_{1 r} e_{l}\right) A Z\right| \\
& =-n|M(\hat{\Lambda})|^{-1}\left|Z_{r}^{\prime}\left(W_{r}\right) A Z\right| \text {. }
\end{aligned}
$$

Parts b) and c) are obtained in a similar way by using parts a) and b) of lemma 3.1 and by recalling the fact that $\partial|M(\Lambda)| /\left.\partial \Lambda\right|_{\Lambda=\hat{\Lambda}}=0$. 
For practical purposes, we get that the set of all $\Lambda$-values such that

$$
(\Lambda-\hat{\Lambda})^{\prime} H(\hat{\Lambda})(\Lambda-\hat{\Lambda}) \leq \chi_{p, \alpha^{\prime}}^{2}
$$

where $H(\hat{\Lambda})$ is the inverse of the matrix in (10), is an approximate $(1-\alpha)_{\mathbf{x}} 100 \%$ confidence ellipsoid for the transformation parameter $\Lambda$. A general expression for the functions $w_{j}^{\left(\lambda_{j}\right)}$ and $U_{j}^{\left(\lambda_{j}\right)}$ can be found in Atkinson and Lawrance (1989).

EXAMPLE 1. A bivariate sample $\left(X_{i}, Y_{i}\right)^{\prime}$ of size $n=50$ is generated through a bivariate lognormal model

$$
(\log X, \log Y)^{\prime} \sim N_{2}\left[\left(\begin{array}{l}
0 \\
0
\end{array}\right) ;\left(\begin{array}{rr}
1 & .5 \\
.5 & 1
\end{array}\right)\right] \text {. }
$$

By minimizing the determinant (5), we get $\hat{\Lambda}=(-.020,-.25)^{\prime}$, with estimated variance-covariance matrix

$$
\left(\begin{array}{ll}
.0197 & .0001 \\
.0001 & .0181
\end{array}\right)
$$

Figure 1 shows the approximate $95 \%$ joint confidence ellipsoid (11) with boundary given by $\chi_{2 ; \alpha}^{2}=5.991$.

Figure 1

The advantage of this approach, in relation to the confidence region (8), is that approximate confidence regions can be explicitly computed. This is important because, when $p \geq 2$, the usual graphical estimation of the transformation parameter, which is extremely useful in the scalar case, is no longer feasible. 


\section{EFFICIENCY CONSIDERATIONS}

Recall that model (2) implies the p marginal models

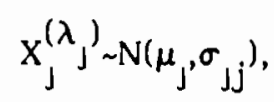

$j=1, \ldots, p$. Model (2) and the family of models (10) are equivalent when the components of the random vector $X=\left(X_{1}, \ldots, X_{p}\right)^{\prime}$ are independent. Andrews et al. (1971) compare the results of fitting by maximum likelihood the model (2) and each of the $p$ marginal models (10) separately and pose the general question if there is something to gain in using the model (2) in relation to the collection of $p$ marginal models (10). A possible answer can be found in the theory what follows.

Define $\hat{\lambda}_{M}=\left(\hat{\lambda}_{1 M}, \ldots, \hat{\lambda}_{\mathrm{PM}}\right)^{\prime}$, where $\hat{\lambda}_{\mathrm{JM}}$ stands for the MLE estimator of $\lambda$, under the jth marginal model. Given that (9) holds, if we can prove that

$$
\hat{\Lambda}_{M} \sim \mathrm{AN}_{\mathrm{P}}\left[\Lambda,(1 / n) \mathrm{J}_{M}(\Lambda, \Lambda)\right]
$$

asymptotic efficiency considerations indicate that the choice between $\hat{\Lambda}$ and $\hat{\Lambda}_{M}$ depends on the relation between the matrices $J(\Lambda, \Lambda)$ and $J_{M}(\Lambda, \Lambda)$. In fact, we have the following theorem.

THEOREM 4.1. Assuming appropriate regularity conditions, we have

a) $\hat{\Lambda}_{M} \sim \mathrm{AN}_{\mathrm{P}}\left[\Lambda,(1 / \mathrm{n}) \mathrm{J}_{\mathrm{M}}(\Lambda, \Lambda)\right]$

b) $J_{M}(\Lambda, \Lambda) \geq J(\Lambda, \Lambda)$, in the sense that the pxp matrix $J_{M}(\Lambda, \Lambda)-J(\Lambda, \Lambda)$ is non negative definite.

PROOF. To simplify the notation, we will make the proof only in the case $p=2$. The ideas in the case of general $p$ are similar. Note that, when $p=2$, the set of parameters under model (2) can be written as $\Theta=\left(\Lambda^{\prime}, \Phi^{\prime}\right)^{\prime}$, $\Phi=\left(\Phi_{1}^{\prime}, \Phi_{2}\right)^{\prime}, \quad$ where $\Phi_{1}=\left(\mu_{1}, \mu_{2}, \sigma_{11}, \sigma_{22}\right)^{\prime} \quad$ and $\Phi_{2}=\sigma_{12}$. Recall that, 
relabelling, $\Theta=(\Lambda, \Phi)=\left(\theta_{1}^{\prime}, \theta_{2}^{\prime}, \sigma_{12}\right)^{\prime}$, where $\theta_{j}=\left(\lambda_{j}, \mu_{j}, \sigma_{j j}\right)^{\prime}, j=1,2$.

a) Let $L_{j}\left(\theta_{j}\right)$ be the log-likelihood of $\theta_{j}$ under the jth marginal model and $\hat{\theta}_{j M}$ the corresponding MLE estimator of $\theta_{j}$. Assuming regularity conditions, we can write

$$
n^{1 / 2}\left(\begin{array}{l}
\hat{\theta}_{1 M^{-}{ }_{1}} \\
\hat{\theta}_{2 M^{-} \theta_{2}}
\end{array}\right)=\left(\begin{array}{cc}
K\left(\theta_{1}\right) & 0_{3 \times 3} \\
0_{3 \times 3} & K\left(\theta_{2}\right)
\end{array}\right)\left(1 / n^{1 / 2}\right)\left(\begin{array}{l}
\partial L_{1}\left(\theta_{1}\right) / \partial \theta_{1} \\
\partial L_{2}\left(\theta_{2}\right) / \partial \theta_{2}
\end{array}\right)+0_{p}(1),
$$

where $K\left(\theta_{1}\right)$ and $K\left(\theta_{2}\right)$ converge in probability to the inverses of the corresponding information matrices $I\left(\theta_{1}\right)$ and $I\left(\theta_{2}\right)$. Therefore, the left hand side of (11) is asymptotically normal with mean zero and variance-covariance matrix given by

$$
\left(\begin{array}{cc}
I\left(\theta_{1}\right)^{-1} & I\left(\theta_{1}\right)^{-1} M\left(\theta_{1}, \theta_{2}\right) I\left(\theta_{2}\right)^{-1} \\
I\left(\theta_{2}\right)^{-1} M\left(\theta_{2}, \theta_{1}\right) I\left(\theta_{1}\right)^{-1} & I\left(\theta_{2}\right)^{-1}
\end{array}\right)
$$

where $\quad \mathrm{M}\left(\theta_{1}, \theta_{2}\right)=\mathrm{E}\left[\left(\partial \mathrm{L}_{1}\left(\theta_{1} / \partial \theta_{1}\right)\left(\partial \mathrm{L}_{2}\left(\theta_{2} / \partial \theta_{2}\right)^{\prime}\right], \quad\right.\right.$ and $\mathrm{M}\left(\theta_{2}, \theta_{1}\right)=\mathrm{M}\left(\theta_{1}, \theta_{2}\right)^{\prime}$. This implies that $n^{1 / 2}\left(\hat{\Lambda}_{M}-\Lambda\right)$ is also asymptotically normal with mean zero and variance-covariance matrix $J_{M}(\Lambda, \Lambda)$ given by the corresponding submatrix of the matrix (12) above.

b) From a standard expansion of the likelihood equation for model (2), we can obtain

$$
\mathrm{n}^{1 / 2}\left(\hat{\sigma}_{12}-\sigma_{12}\right)=\mathrm{K}\left(\theta_{1}, \theta_{2}, \sigma_{12}\right)\left(1 / \mathrm{n}^{1 / 2}\right)(\partial \mathrm{L} / \partial \theta)+\mathrm{o}_{\mathrm{p}}(1),
$$

where $K\left(\theta_{1}, \theta_{2}, \sigma_{12}\right)$ is a $1 \times 7$ matrix which converges in probability to the corresponding submatrix of the inverse of the information matrix under model (2). By adjoining expansions (11) and (13) we get that $\mathrm{n}^{1 / 2}\left[\left(\hat{\theta}_{1 \mathrm{M}}-\theta_{1}\right)^{\prime},\left(\hat{\theta}_{2 \mathrm{M}}-\theta_{2}\right)^{\prime}, \hat{\sigma}_{12}-\sigma_{12}\right]^{\prime} \quad$ is asymptotically normal with mean zero and variance-covariance matrix $V(\Lambda, \Phi)$, say. By the asymptotic optimality of the MLE estimator of $(\Lambda, \Phi)$ under model (2), we get that 
$V(\Lambda, \Phi) \geq I(\Lambda, \Phi)^{-1}$ and the same is true for the submatrices which correspond to $\Lambda$. Therefore, $J_{M}(\Lambda, \Lambda) \geq J(\Lambda, \Lambda)$.

As a conclusion, the estimates obtained by fitting each of the marginal models separately are less efficient than the joint MLE $\hat{\Lambda}$. As the following examples show, the loss of efficiency might be severe and , therefore, $\hat{\Lambda}$ is preferable to $\hat{\Lambda}_{\mathrm{M}}$.

EXAMPLE 2. Consider the case $p=2$ and $\Lambda=(0,0)^{\prime}$. After lengthy algebra, it can be shown that

$$
J_{M}(\Lambda, \Lambda)=D^{1 / 2}\left(\begin{array}{cc}
1 ; & \rho^{3} \\
\rho^{3} ; & 1
\end{array}\right) D^{1 / 2},
$$

where $D=\operatorname{diag}\left(2 /\left(3 \sigma_{11}\right), 2 /\left(3 \sigma_{22}\right)\right)$, and $\rho$ is the correlation coefficient between $\log X$ and $\log Y$. We also obtain

$$
J(\Lambda, \Lambda)=D^{1 / 2}\left(\begin{array}{ll}
P_{11}(\rho) ; & P_{12}(\rho) \\
P_{12}(\rho) ; & P_{22}(\rho)
\end{array}\right) D^{1 / 2},
$$

where $\mathrm{p}_{11}(\rho)=\mathrm{p}_{22}(\rho)=\left[1+\left(\left[\rho^{2}\left(\rho^{2}+3\right)\right] /\left[3\left(3-2 \rho^{2}\right)\right]\right)\right]^{-1}$ and $\mathrm{p}_{12}(\rho)=$ $\rho^{3}\left[1+(1 / 3)\left(\rho^{4}-3 \rho^{2}+6\right)\right]^{-1}$. Note that the diagonal entries of the matrix $\mathrm{J}_{M}(\Lambda, \Lambda)$, namely, $2 /\left(3 \sigma_{11}\right)$ and $2 /\left(3 \sigma_{22}\right)$, agree with the expression of the asymptotic variance for $\hat{\lambda}_{J M}$ in the case of $\lambda_{j}=0$, which was obtained by Hinkley (1975).

It is easily shown that the matrix $J_{M}(\Lambda, \Lambda)-J(\Lambda, \Lambda)$ equals to zero when $\rho=0$ and is positive definite for $0<|\rho|<1$. For $|\rho|<1$, the asymptatic relative efficiency (ARE) of the MLE $\hat{\Lambda}$ with respect to $\hat{\Lambda}_{M}$ is given by (Serfling (1980), p. 141),

$$
\operatorname{ARE}\left(\hat{\Lambda}, \hat{\Lambda}_{M}\right)=\left[|J(\Lambda, \Lambda)| /\left|J_{M}(\Lambda, \Lambda)\right|\right]^{1 / 2}
$$




$$
=\frac{3}{\left[\left(1+\rho^{2}+\rho^{4}\right)\left(\rho^{4}-3 \rho^{2}+9\right)\right]^{1 / 2}} .
$$

Table 1 displays the value of the ARE for selected values of $\rho$. Recall that the ARE is bounded between $1(\rho=0)$ and $(3 / 7)^{1 / 2}=.655$ which is the limiting value as $|\rho|$ approaches 1 .

\begin{tabular}{c|ccccccccc}
$\rho$ & .00 & .25 & .33 & .50 & .75 & .90 & .95 & .975 & .99 \\
\hline ARE & 1.000 & 0.978 & 0.908 & 0.792 & 0.738 & 0.710 & 0.683 & 0.668 & 0.660
\end{tabular}

Table 1. ARE $\left(\hat{\Lambda}, \hat{\Lambda}_{M}\right)$ for selected values of $\rho$.

EXAMPLE 3. Let $(X, Y)^{\prime}$ be a bivariate random vector. Consider estimation of the scalar parameter $\lambda$ from two different models:
a) (Joint mormality)
$\left(X^{(\lambda)}, Y\right)^{\prime} \sim N_{2}(\mu, \Sigma)$;
b) (Marginal normality)
$\mathrm{X}^{(\lambda)} \sim \mathrm{N}\left(\mu_{1}, \sigma_{11}\right)$.

Let $\hat{\lambda}$ be the MLE of $\lambda$ under model a) and let $\hat{\lambda}_{M}$ be the MLE of $\lambda$ under model b). If $\operatorname{av}\left[\hat{\lambda}_{M}\right]$ and $\operatorname{av}[\hat{\lambda}]$ are the respective asymptotic variances of $\hat{\lambda}_{M}$ and $\hat{\lambda}$, it can be shown that, when $\lambda=0$,

$$
\operatorname{av}\left[\hat{\lambda}_{M}\right]=\operatorname{av}[\hat{\lambda}]\left[1+\frac{\rho^{2}}{3\left(1-\rho^{2}\right)}\right] \text {. }
$$

for $|\rho|<1$. From (17), the two asymptotic variances are the same when $\rho=0$. However, the loss of efficiency can be very large for values of $|\rho|$ close to one.

\section{APPENDIX}

PROOF OF LEMMA 3.1. We will proof only part a) since parts b) and c) are 
similar. Write $C=\left(c_{1}, \ldots, c_{p}\right), \quad D=\left(d_{1}, \ldots, d_{p}\right)$ and let $Q=\left(q_{r s}\right)=C^{\prime} E D$. Therefore $q_{r s}=c_{r}^{\prime} E d_{s}$. Standard results on matrix differentiation show that $\partial|Q| / \partial q_{r s}=Q_{r s}$, where $Q_{r s}$ is the cofactor of the element $q_{r s}$ (see, for example, Mardia et al. (1979, p.479)). By the chain rule we have

$$
\begin{gathered}
\partial\left|C^{\prime} E D\right| / \partial c_{i j}=\sum_{r=1 s=1}^{p} \sum_{s=1}^{p}\left(\partial|Q| / \partial q_{r s}\right)\left(\partial q_{r s} / \partial c_{1 j}\right)=\sum_{s=1}^{p} Q_{j s}\left(\sum_{\beta=1}^{n} e_{1 \beta} d_{\beta s}\right) \\
=\left|C_{j}^{\prime}\left(e_{1}\right) E D\right| .
\end{gathered}
$$

\section{REFERENCES}

ANDREWS, D. F., GNANADESIKAN, R.\& WARNER, J.L. (1971). Transf ormations of multivariate data. Biometrics, 27, 825-840.

ATKINSON, A.C. \& LAWRANCE, A.J. (1989). A comparison of asymptotically equivalent test statistics for regression transformation. Biametrika 76, 223-229.

BOX, G.E.P.\& COX, D.R. (1964). An analysis of transformations. Laurnal of the Royal Ptatistical Yociety, Ser. B., 26, 211-52.

GNANADESIKAN, R. (1977). Methods for Ytatistical Data Analysis of Multivariate Observations. (J. Wiley: New York).

HINKLEY, D.V. (1975). On power transformations to symmetry. Biametrika, 62, 101-111.

MARDIA, K.V., KENT, J.L. \& BIBBY, J.M. (1979). Multivariate Analysis. (Academic Press: London).

SEBER, G.A.F. (1984). Multivariate Observations. (J. Wiley: New York).

SEBER, G.A.F. \& WILD, C.J. (1989). Nonlinear Regressian. (J. Wiley: New York). 
SERFLING, R.J. (1980). sppraximation Thearems of Mathematical Statistics. (J. Wiley: New York). 


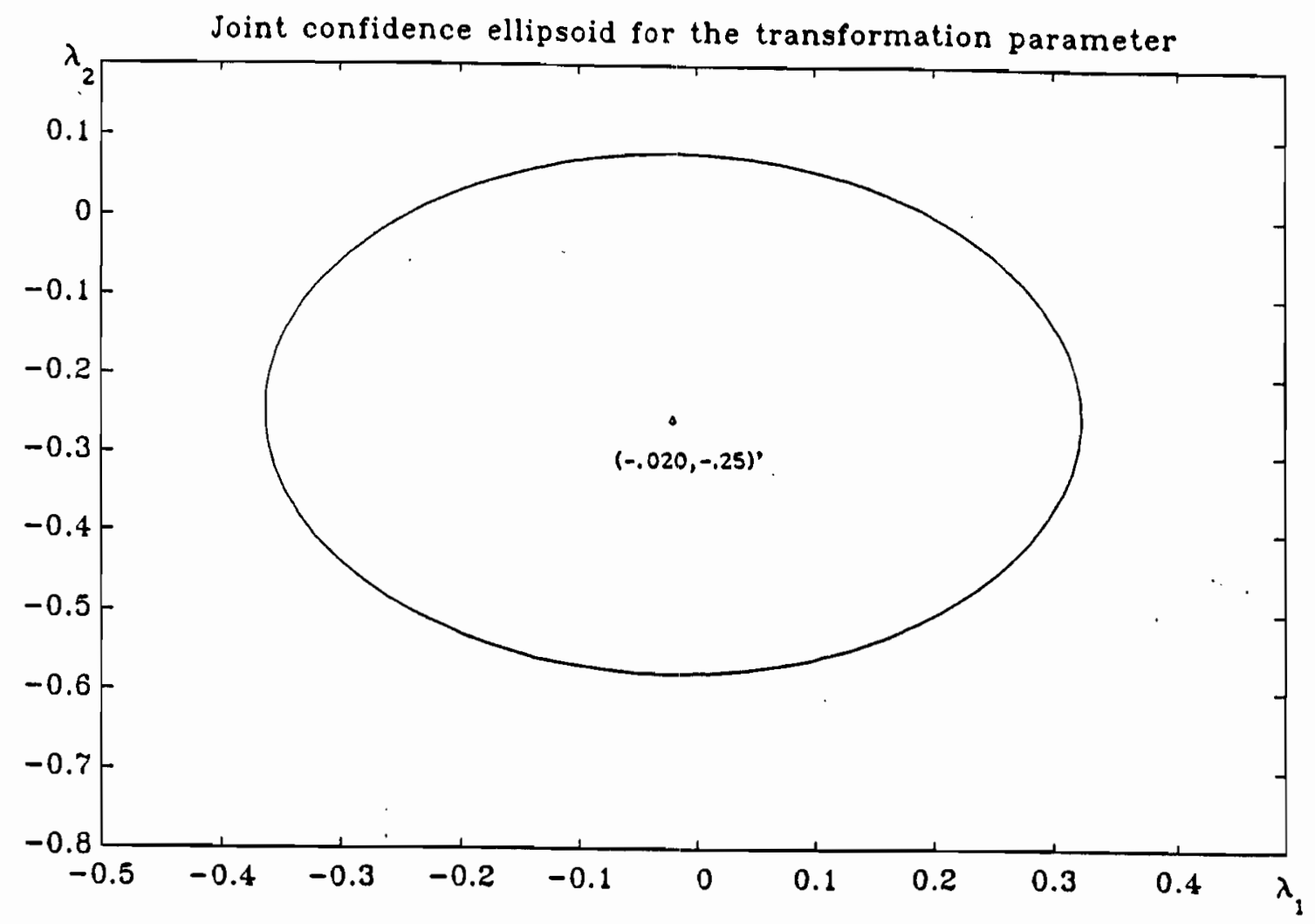

Figure 1. Confidence ellipsoid for $\Lambda=\left(\lambda_{1}, \lambda_{2}\right)$. 